University of New Hampshire

University of New Hampshire Scholars' Repository

$9-1996$

\title{
In-Situ Far-Field Calibration of Multibeam Sonar Arrays for Precise Backscatter Imagery
}

\author{
Larry Fusillo \\ Computer Sciences Corporation, Moorestown, NJ \\ Christian de Moustier \\ University of California - San Diego \\ John H. Satriano \\ Naval Command Control \& Ocean Surveillance Center \\ Stanley Zietz \\ Drexel University
}

Follow this and additional works at: https://scholars.unh.edu/ccom

Part of the Oceanography and Atmospheric Sciences and Meteorology Commons

\section{Recommended Citation}

Fusillo, Larry; de Moustier, Christian; Satriano, John H.; and Zietz, Stanley, "In-Situ Far-Field Calibration of Multibeam Sonar Arrays for Precise Backscatter Imagery" (1996). IEEE Oceans. 192.

https://scholars.unh.edu/ccom/192

This Conference Proceeding is brought to you for free and open access by the Center for Coastal and Ocean Mapping at University of New Hampshire Scholars' Repository. It has been accepted for inclusion in Center for Coastal and Ocean Mapping by an authorized administrator of University of New Hampshire Scholars' Repository. For more information, please contact Scholarly.Communication@unh.edu. 


\section{In-Situ Far-Field Calibration Of Multibeam Sonar Arrays For Precise Backscatter Imagery}

\author{
Larry Fusillo \\ Computer Sciences Corporation \\ Moorestown NJ 08057
}

John H Satriano

Naval Command Control \& Ocean Surveillance

Center

Warminster PA 18974

\author{
Christian de Moustier \\ Marine Physical Laboratory \\ Scripps Institution of Oceanography \\ La Jolla CA 92093
}

\author{
Stanley Zietz \\ Drexel University \\ Philadelphia PA 19104
}

\begin{abstract}
Calibration of large hull-mounted sonar transducer arrays is usually done by measuring the performance of individual components in a test tank. Multiple transducer element beam patterns are superimposed and combined with window attenuation and other partial measurements to give an approximation of the total beam pattern. This process often ignores or fails to accurately model factors such as mounting hardware, array misalignment, reflections from the hull structure and other real world absorbers, reflectors and attenuators which can have significant effect on the actual beam pattern. This paper describes an application of a novel technique for in-situ measurement of the far-field beam pattern of an entire installed hydrophone array using an ROV to carry a calibrated reference transducer.
\end{abstract}

The sonar array is part of a Simrad EM-121 multibeam sonar system installed aboard the Navy's new survey ship the USNS SUMNER TAGS-61. Test tank measurements of individual hydrophone transducer packages were available and a composite array beam pattern was computed from this data. Direct at-sea measurement of the far-field beam pattern was then made by placing a reference transducer on an ROV and navigating it through an arc in the far field of the shipboard array. These two sets of beam patterns are compared

Backscattered signals in a multibeam sonar system can be the basis for characterization of sea floor morphology in terms of mud, clay, silt, sand, gravel, rocks, etc. The degree of differentiation between bottom types, or the number of bottom types which can be differentiated, depends on how accurately the signal strength is calibrated. Factors which affect this measurement include the sonar transmitter electrical output, projector efficiency, projector array beam pattern, spreading loss and attenuation in the water column, losses at the bottom reflection, hydrophone array beam pattern, hydrophone sensitivity and receiver gains. The degree of improvement in sea floor characterization resulting from the in-situ hydrophone array calibration will be examined.

\section{Introduction}

This report presents the results of a series of measurements carried out at sea aboard the Navy's new oceanographic research vessel, USNS SUMNER, TAGS-61 in December 1995. The work involved collaborative efforts of personnel from Computer Sciences Corporation, the Naval Command Control and Ocean Surveillance Center (NRaD), the Marine Physical Laboratory of Scripps Institution of Oceanography, Drexel University and the Naval Oceanographic Office.

Measurements were made to determine the hydrophone array beam pattern of the SIMRAD EM-121 multibeam echo-sounder installed on the SUMNER. The EM-121 multibeam sonar operates at an acoustic frequency of $12 \mathrm{kHz}$ and is designed for sounding over a 120 degree wide swath in water depths from 10 to 11,000 meters. The hydrophone array consists of 128 hydrophone staves, each 0.54 meters long and spaced .058 meters apart. The array is divided into two sections and mounted athwartships in a $\mathrm{V}$ configuration. The sides of the $\mathrm{V}$ are inclined 10 degrees from horizontal, with 64 hydrophone staves on each side of the keel. The far-field for one side of the $V$ array is approximated (using the ratio of the square of the array aperture to the sonar frequency) to be 107 meters. 


\section{Conventional Beam Pattern Plot}

Transducer beam patterns were provided by the manufacturer for each of the individual hydrophones. The physical limitations of test tanks and equipment as well as the need to operate within the transducer's far field make it impractical to perform these measurements on a complete array. Therefore, it is necessary to measure individual transducers and combine the results into an estimate of the full array pattern. It is also necessary to ignore the effects of array windows, mounting systems and the large reflecting surfaces of the ship's hull and the ocean surface. Fig.1 shows a composite beam pattern for the EM-121 array constructed by averaging measured beam patterns for sixteen representative hydrophones.

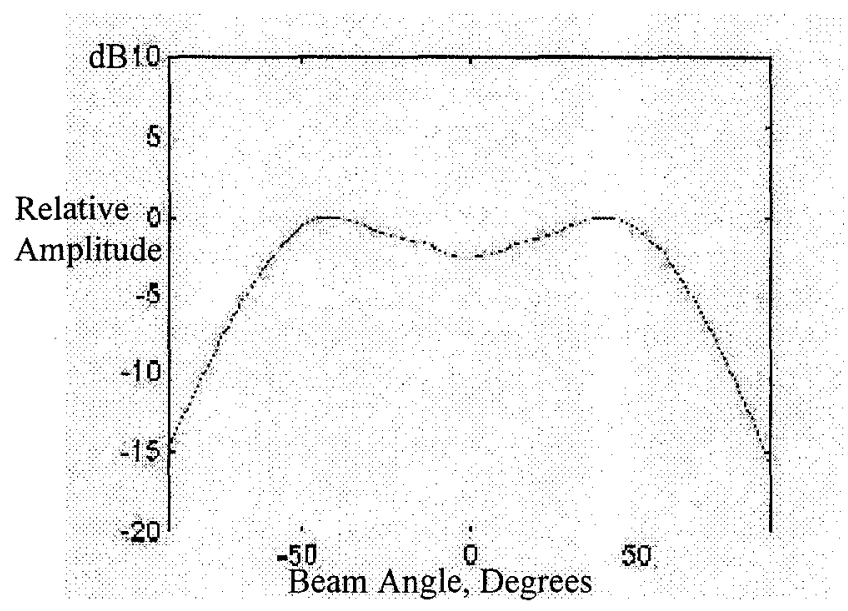

Fig. 1. Composite beam pattern of hydrophone array based on test tank measurements of individual transducers.

\section{Test Equipment Setup}

The Phantom DS4 ROV was provided by Scripps Institute of Oceanography. The DS4 ROV has 4 horizontal thrusters and two dihedral thrusters for maneuvering. An ITC-1007 $12 \mathrm{kHz}$ calibrated acoustic transducer was mounted on a fiberglass pole extending 1 meter from the ROV. The ROV was navigated using an ORE Intl. Trackpoint II ultrashort baseline navigation system. The ITC-1007 was used as a 12 $\mathrm{kHz}$ sound source, emitting a $100 \mathrm{msec}$ pulse once every 6 seconds.

\section{Test Operations}

The ROV was tethered to its shipboard control and data recording station as shown in Fig. 2 . The first $100 \mathrm{~m}$ of this cable was tied to the ship's oceanographic winch cable which was held vertical by a weight. An additional $100 \mathrm{~m}$ of free cable allowed the ROV to roam. This set the operating depth of the ROV to $100 \mathrm{~m}$ with a scope of $100 \mathrm{~m}$. During the calibration tests. the ROV was lowered to its operating depth, then maneuvered to position its transducer on a path across the beam pattern of the shipboard array.

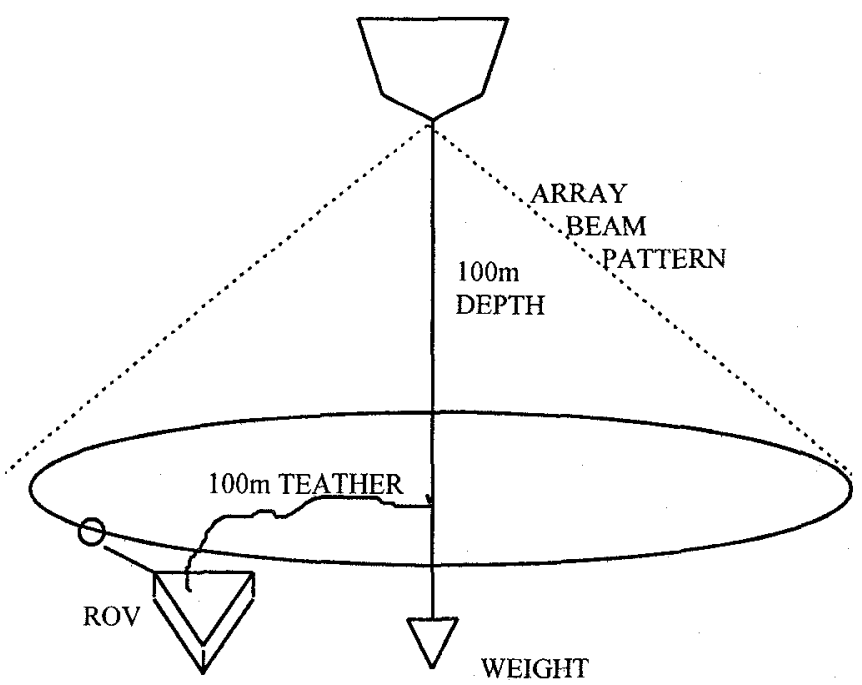

Fig. 2. Operating configuration.

\section{Data Collection}

The signals from the ROV's transducer were received at the EM-121 hydrophone array and was sampled and digitized into in-phase and quadrature components at a sampling interval of 400 usec. Software was developed to record this data on hard disk using the NRaD Geoacoustic Echo Processor (GEP). The data was then transferred notebook PC which used a MathCad software package to providing atsea viewing to verify data integrity. Data was collected in 5 minute blocks with the ROV traversing an arc on both the port and starboard sides of the ship.

\section{Signal Processing}

The angle between the hull-mounted array and the point of origination of the signal at the ROV was determined using a spatial fast fourier transform technique which examines the phase distribution across the array. The FFT's were performed on each side of the V array separately. The FFT's were performed using Matlab software on a desktop 80486 PC. Each FFT yielded a single peak corresponding the beam arrival angle. Thus, the bearing arrival angle was 
determined directly from the hydrophone measurements with reference to the transponder navigation. (Errors in signal strength due to variations in range to the ROV were considered small and were ignored in this preliminary processing.)

The FFT peaks provided both bearing arrival angle and sonar signal amplitude. These points are plotted in Fig.3. Each dot represents a 400 usec sample in amplitude vs angle. This figure compares the at-sea measurements with the test tank curve of Fig. 1. As can be seen, both techniques produces beam patterns with similar characteristics, including the $3 \mathrm{~dB}$ dip at the center of the hydrophone response.

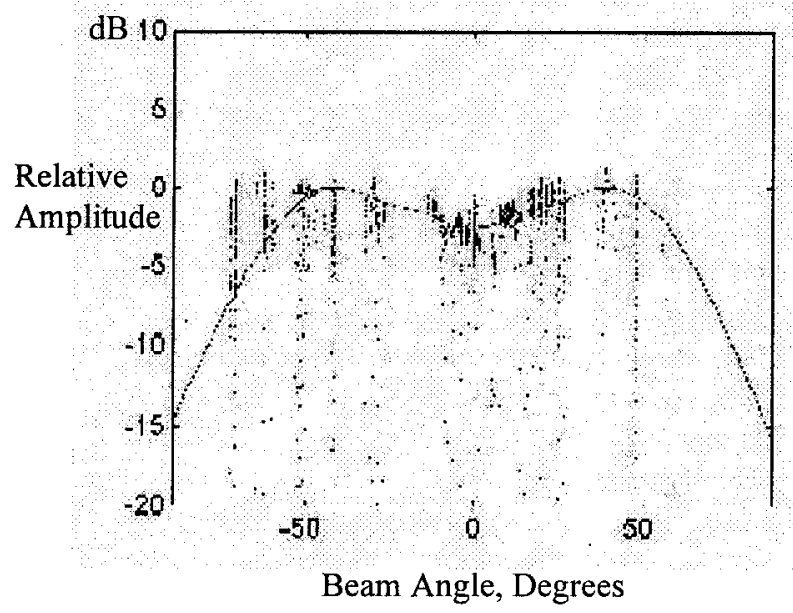

Fig. 3. At-sea array beam pattern measurements compared to test tank plot.

The dense band of at-sea points at the top is from the flat portion of the $100 \mathrm{msec}$ transmitted pulse, while the "noise" below is due to samples occurring during the rise time when the array was only partially illuminated. Fig. 4 shows the data samples for a single $100 \mathrm{msec}$ ping. In this figure, a small number of samples are present during the rise and fall times, while the responses during the steady-state part of the pulse are nearly equal. For improved accuracy, the rise and fall time samples should be deleted from the data files.

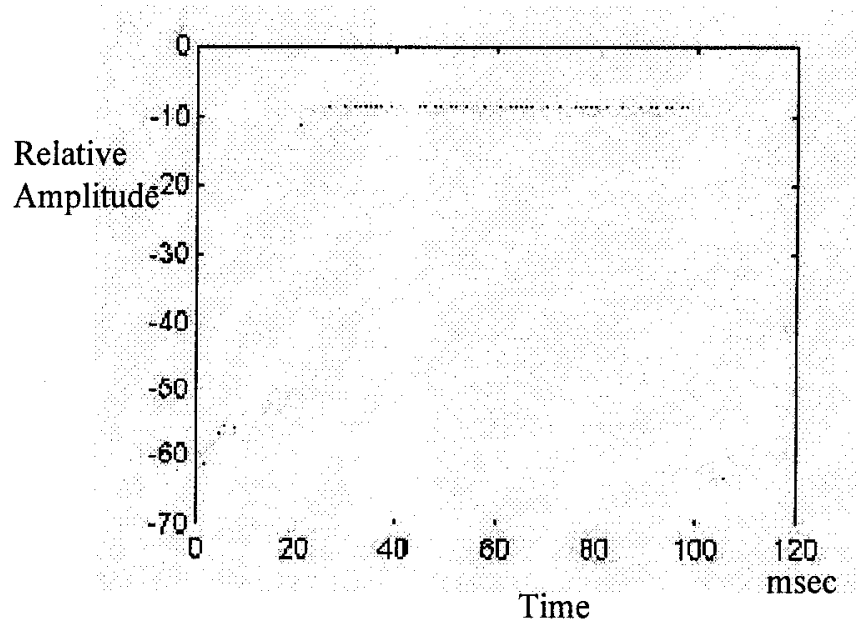

Fig. 4. Typical data samples for a single transmitted pulse.

The variability of the returns from the ITC-1007 sound source on the flat parts of the pulse returns were within $+/$ $1.5 \mathrm{~dB}$ three sigma around the average curve of 16 hydrophone test tank measurements. Some of the variability is attributable to the rise time sampling of each pulse with the remainder due to environmental conditions. Wind speed was 25-30 knots and sea-state was 4-5 for the data collection period. Because the flat part of the pulse return varied by less than $0.3 \mathrm{~dB}$ within each 5 minute sample, we expect that it is possible to obtain in-situ hydrophone beam pattern measurements to within $+/-0.1 \mathrm{~dB}$ one sigma when using a calibrated sound source, a good navigation system and a when conducting the tests in a low sea-state.

\section{Conclusions}

The ROV method for measuring beam patterns of large sonar arrays has been successfully tested. The measured beam pattern shows good agreement with the test tank version in form and amplitude, including reproduction of the axial dip. However, there are interesting differences between the two results, such as the significantly higher response at the outer beams; this may be an effect of reflections from the ship's hull. Further refinement of our signal processing technique and inclusion of additional data samples can be expected to clarify this proposition. 